\title{
Direct radiolabeling of nivolumab with Ga-68: A novel PET tracer to detect PD-1 expressing tumors
}

Silvia Migliari ${ }^{*}$, Antonino Sammartano ${ }^{1}$, Benedetta Pellegrino ${ }^{2}$, Davide Cavazzini ${ }^{3}$, Simone Ottonello ${ }^{3}$, Gabriele Missale ${ }^{4}$, Antonino Musolino $^{2}$ and Livia Ruffini ${ }^{1}$

${ }^{1}$ Nuclear Medicine and Molecular Imaging Department, University Hospital of Parma, via Gramsci 14, 43126 Parma, Italy

${ }^{2}$ Medical Oncology Unit, University Hospital of Parma, via Gramsci 14, 43126 Parma, Italy

${ }^{3}$ Department of Chemical and Life Sciences, University of Parma, Parco Area delle Scienze, 11/a - 43124 Parma, Italy

${ }^{4}$ Laboratory of Viral Immunopathology, University Hospital of Parma, via Gramsci 14, 43126 Parma, Italy

In recent years, the blockade of immune checkpoint molecules with monoclonal antibodies, like those targeting the PD-1/PD-L1 pathway, has enabled the development of breakthrough therapies in oncology, leading to delayed tumor growth and increased survival.

Non-invasive methods permitting whole-body detection of PD-1 and PD-L1 at high sensitivity and resolution could thus be highly useful in-patient selection and monitoring of PD-1/PD-L1 expression during disease progression and treatment, therefore different approaches have been developed in order to label tumor-specific monoclonal antibodies (Mo)Abs with PET and SPECT radioisotopes.

Aim of this study was to develop an immunoPET tracer for imaging PD-1 using Nivolumab (BMS-936558, ONO-4538, or MDX1106, trade name Opdivo; Bristol-Myers Squibb, Princeton, NJ, USA), the firstin-human immunoglobulin G4 (IgG4) PD-1 immune checkpoint inhibitor antibody, and the PET radioisotope Gallium-68.

Direct labeling approach procedure involves the use of a solution to buffer the acidity of the eluate ${ }^{68} \mathrm{GaCl}_{3}$ of a commercially avaible $\mathrm{TiO}_{2}$ based ${ }^{68} \mathrm{Ge} /{ }^{68} \mathrm{Ga}$ generator (Eckert \& Ziegler, E\&Z, Berlin, Germany) in order to prepare the carboxylic and aminic groups of Nivolumab that can be conveniently used as coordinating sites to bind Gallium-68.

Copyright: (C2017 Migliari S. This is an open-access article distributed under the terms of the Creative Commons Attribution License, which permits unrestricted use, distribution, and reproduction in any medium, provided the original author and source are credited.
The ${ }^{68} \mathrm{Ge} /{ }^{68} \mathrm{Ga}$ generator was eluted with $10 \mathrm{ml} 0.1 \mathrm{M} \mathrm{HCl}$ following the manufacture's protocol.

A solution of ultrapure $\mathrm{NaOAc} 1.25 \mathrm{M}$ (Fluka Traceselect, $\geq 99.99 \%$, metal basis) was added to this $0.1 \mathrm{M} \mathrm{HCl}$ solution of $\left[{ }^{68} \mathrm{Ga}\right] \mathrm{Ga}$-Chloride bringing the $\mathrm{pH}$ to $4.5-5$. The resulting $\left[{ }^{68} \mathrm{Ga}\right] \mathrm{Ga}$-acetate solution (ca. $50-100 \mathrm{MBq})$ was added to Nivolumab $(10 \mathrm{mg} / \mathrm{ml})$ protein solution.

The $\left[{ }^{68} \mathrm{Ga}\right] \mathrm{Ga}$-Nivolumab solution was incubated in a heat block at $45^{\circ} \mathrm{C}$ for 40 minutes. The resulting $\left[{ }^{68} \mathrm{Ga}\right] \mathrm{Ga}$-Nivolumab was isolated by centrifugation and the radiolabeling yield has been calculated.

The radiochemical purity of $\left[{ }^{68} \mathrm{Ga}\right] \mathrm{Ga}$-Nivolumab was determined using instant thin layer chromatography (TLC): TLC-SG strips are used as stationary phase and ammonium acetate (sol aq. 10\%) : $\mathrm{MeOH}$ (1:1) as mobile phase to separate free Gallium-68, which remains at the bottom, while the radiolabelled (Mo)Abs moved to the top.

The promising labeling results show an efficient and rapid direct procedure to label (Mo)Abs with Gallim-68 reducing reaction steps and antibody preparation and allowing the future formulation of freeze-dried kit to obtain a PET imaging of PD-1 expressing tumors.
Correspondence to: Silvia Migliari, Nuclear Medicine and Molecular Imaging Department, University Hospital of Parma, via Gramsci 14, 43126 Parma, Italy, E-mail: smigliari@ao.pr.it

Received: October 04, 2017; Accepted: October 19, 2017; Published: October 23,2017 